\title{
Interference phenomena, chiral bosons, and Lorentz invariance
}

\author{
E. M. C. Abreu* and A. de Souza Dutra ${ }^{\dagger}$ \\ Departamento de Física e Química, Universidade Estadual Paulista, Av. Ariberto Pereira da Cunha 333, Guaratinguetá, 12516-410, \\ São Paulo, SP, Brazil
}

(Received 20 December 2000; published 28 June 2001)

\begin{abstract}
We have studied the theory of gauged chiral bosons and proposed a general theory, a master action, that encompasses different kinds of gauge field couplings in chiral bosonized theories with first-class chiral constraints. We have fused opposite chiral aspects of this master action using the soldering formalism and applied the final action to several well-known models. The Lorentz rotation permitted us to fix conditions on the parameters of this general theory in order to preserve the relativistic invariance. We also have established some conditions on the arbitrary parameter concerned in a chiral Schwinger model with a generalized constraint, investigating both covariance and Lorentz invariance. The results obtained supplement the one that shows the soldering formalism as a new method of mass generation.
\end{abstract}

DOI: 10.1103/PhysRevD.64.025023

PACS number(s): 11.10.Ef, 04.65.+e

\section{INTRODUCTION}

The research in chiral bosonization began many years back with the seminal paper of Siegel [1]. Floreanini and Jackiw offered some different solutions to the problem of a single self-dual field [2]. The study of chiral bosons has blossomed thanks to the advances in superstring compactification [3] and in the construction of interesting theoretical models, such as the Thirring model [4]. They also play an important role in the studies of the quantum Hall effect [5]. The introduction of a soliton field as a charge-creating field obeying one additional equation of motion leads to a bosonization rule [6]. In the course of the analysis of the chiral boson properties, one natural step is to couple them to Abelian and non-Abelian gauge fields $[7,8]$ in order to study the correspondent anomalies, or to provide an alternative approach to chiral models in two dimensions [9]. Gates and Siegel showed how to construct general interacting actions for chiral bosons, including the supersymmetric and the nonAbelian cases [10]. They used this construction to obtain the righton-lefton interaction by carrying out the path-integral quantization in a generalized Thirring model. Other couplings of the chiral bosons with supersymmetry and gravity can be found in Ref. [11]. In an alternative way, Stone [12] has shown that the method of coadjoint orbit, when applied to a representation of a group associated with a single affine Kac-Moody algebra, generates an action for the chiral WessZumino-Witten (WZW) model [13], a non-Abelian generalization of the Floreanini and Jackiw (FJ) model. The formalism introduced by Stone has been interpreted recently as a new method of mass generation [14].

In the context of chiral theories in two dimensions, Harada has shown [9] how to obtain a consistent coupling of FJ chiral bosons with a $\mathrm{U}(1)$ gauge field, starting from the chiral Schwinger model and discarding the right-handed degrees of freedom by means of a projection in phase space implemented by the chiral constraint $\pi_{\phi}=\phi^{\prime}$. Later on, it

\footnotetext{
*Email address: everton@feg.unesp.br

${ }^{\dagger}$ Email address: dutra@feg.unesp.br
}

was observed that starting with a chiral Schwinger model of a given chirality it is possible to couple chiral bosons to $\mathrm{U}(1)$ gauge fields in two Lorentz invariant ways, using different chiral constraints $[15,16]$. The theory proposed was shown to be equivalent to a specific coupling of Siegel's chiral bosons with $\mathrm{U}(1)$ gauge fields which is symmetric under chiralitypreserving gauge transformations.

In Ref. [8], Bellucci, Golterman, and Petcher introduced an $\mathrm{O}(N)$ generalization of Siegel's model for chiral bosons coupled to Abelian and non-Abelian gauge fields. The physical spectrum of the resulting Abelian theory is that of a (massless) chiral boson and a free massive scalar field. Bazeia [17] showed that the Bellucci et al. model is equivalent, at the classical level, to the gauged FJ chiral boson found by Harada.

In this work we have proposed a general action to describe the gauge coupling in different chiral bosonization schemes. We used the soldering formalism proposed by Stone [12] to fuse opposite chiralities producing a final action which was used to apply to various chiral theories with new outcomes. We have also analyzed the problem of the Lorentz invariance of this general model. In Ref. [15], a bosonized form of the chiral Schwinger model with a generalized constraint was analyzed using the Lorentz rotation to fix a general parameter. The soldering formalism permitted us to fix conditions on this parameter to obtain manifest covariance or, in another case, Lorentz invariance of this selfdual action.

We have organized the paper in the following way: in Sec. II we have tried to make a self-consistent review of the soldering formalism and used the well-known Siegel's theory as an example to clarify the interference concepts. In Sec. III we have introduced a general action, a master action, which encompasses different gauged self-dual actions with first-class constraints. The technique of soldering has been applied and the final soldered action was used in several models with new results. In Sec. IV we have employed the Lorentz rotation to fix the value of the parameters in order to guarantee the relativistic invariance of the theory. In Sec. V the interference effect has been analyzed using the chiral Schwinger model with a generalized constraint. The param- 
eter dependence was placed in the light of the manifest covariance and of the Lorentz invariance of the soldered action. The conclusions are depicted in Sec. VI.

\section{REVIEW OF THE SOLDERING FORMALISM}

In this section we will follow basically Refs. [18] and [19] to make a short, but at the same time self-consistent, review of the method of soldering two opposite chiral versions of a theory.

The soldering formalism gives a useful bosonization scheme for Weyl fermions, since a level one representation of $\mathrm{LU}(N)$ has an interpretation as the Hilbert space for a free chiral fermion [20]. However, only Weyl fermions can be analyzed in this way, since a 2D conformally invariant quantum field theory (QFT) has separated right and left current algebras. In other words, it is trivial to make a (free) Dirac fermion from two (free) Weyl fermions with opposite chiralities. The action is just the sum of two Weyl fermion actions. It seems, however, nontrivial to get the action of the WZW model from two chiral boson actions of opposite "chiralities," because it is not the sum of the two.

To solve this problem, Stone [12] introduced the idea of soldering the two chiral scalars by introducing a nondynamical gauge field ${ }^{1}$ to remove the degree of freedom that obstructs the vector gauge invariance [18]. This is connected, as we said above, to the necessity that one must have more than the direct sum of two fermion representations of the Kac-Moody algebra to describe a Dirac fermion. In another way we can say that the equality for the weights in the two representations is physically connected with the necessity to abandon one of the two separate chiral symmetries, and accept that the vector gauge symmetry should be maintained. This is the main motivation for the introduction of the soldering field which makes possible the fusion of dualities in all space-time dimensions. Besides, being just an auxiliary field, it may posteriorly be eliminated in favor of the physically relevant quantities. This restriction will force the two independent chiral representations to belong to the same multiplet, effectively soldering them together. We will see below, in a precise way, more details about the physical significance of the soldering field.

It is worth mentioning that the soldering procedure has a typical quantum-mechanical nature, with no classical analogue. It makes no sense to sum two classical actions describing opposite aspects of some (duality) symmetry that depend on the same field. On the other hand, the direct sum of duality symmetric actions depending on different fields would not give anything new. It is the soldering process that leads to a new and nontrivial result.

In Ref. [19], the authors have promoted soldering the two (Siegel) invariant representations of opposite chiralities. The symmetry content of each theory is well described by the Siegel algebra, a truncate diffeomorphism, that disappears at the quantum level. The resulting action is invariant under the

\footnotetext{
${ }^{1}$ In Ref. [21], Harada proposed a physical interpretation for these soldering fields.
}

full diffeomorphism group, which is not a mere sum of two Siegel symmetries. As we will see later, the result can also be seen as a scalar field immersed in a gravitational background.

Recently, there has been a great deal of interest in soldering together distinct manifestations of duality. The procedure leads to new physical results including quantum contributions. For instance, these results provided the idea of an interference effect. However, this "wave" interpretation is not new. Witten, in Ref. [13], associated the fields depending on only one chirality to left-moving or right-moving waves as being the $\gamma^{5}$ eigenstates.

One of us, with collaborators [14], has promoted the interference of two chiral Schwinger models with opposite chiralities. As a result a new method of mass generation was obtained. The Bose symmetry fixed the Jackiw-Rajaraman parameter $(a=1)$ [22] so that in the spectrum only massless harmonic excitations have survived. The soldered action represents a vector Schwinger model which has a massive particle spectrum. This behavior characterizes a constructive interference with the arising of a mass term that is typical of the right-left quantum interference [23]. ${ }^{2}$

In terms of degrees of freedom we can say that each (chiral) action contributes with "one-half" degree of freedom of opposite signals. Hence the soldered action has one degree of freedom. By the way, in Ref. [25] it was shown that the direct sum of two chiral Schwinger models (CSM) with opposite chiralities is, in fact, equivalent to a sum of a vector Schwinger model (VSM) and an axial Schwinger model (ASM), so, getting a different number of degrees of freedom from a sum of isolated CSM.

It was shown lately [26] that in the soldering process of two Siegel's [1] modes (lefton and righton) coupled to a gauge field [10], this gauge field has decoupled from the physical field. The final action describes a nonmover field (a noton) at the classical level. The noton acquires dynamics upon quantization. This field was introduced by Hull [27] to cancel out the Siegel anomaly. It carries a representation of the full diffeomorphism group, while its chiral components carry the representation of the chiral diffeomorphism.

In the 3D case, the soldering mechanism was used to show the result of fusing together two topologically massive modes generated by the bosonization of two massive Thirring models with opposite mass signatures in the longwavelength limit. The bosonized modes, which are described by self- and anti-self-dual Chern-Simons models [28,29], were then soldered into the two massive modes of the 3D Proca model [30]. In the 4D case, the soldering mechanism produced an explicitly dual and covariant action as the result of the interference between two Schwarz-Sen [31] actions displaying opposite aspects of the electromagnetic duality [30].

Wotzasek [32] has obtained the field theoretical analogue of the "quantum destructive interference" phenomenon, by coupling the non-Abelian chiral scalars to appropriately trun-

\footnotetext{
${ }^{2}$ The extension of this case to the four-dimensional one was performed in Ref. [24].
} 
cated metric fields, known as chiral WZW models, or nonAbelian Siegel models [13]. In fact, this effective action does not contain either right or left movers, but can be identified with the non-Abelian generalization of the bosonic nonmover action proposed by Hull.

In a recent work [33], Abreu et al. analyzed the restrictions posed by the soldering formalism over a new regularization class that extends the classification of the regularization ambiguity of a 2D fermionic determinant from a threeto a four-constraint class. This analysis results from the interference effects between right and left movers, producing a massive vectorial photon that constrains the regularization parameter to this four-constraint class. In other words, the new Faddeevian class of chiral bosons proposed by Mitra [34] has interfered constructively to produce a massive vectorial mode.

The basic idea of the soldering procedure is to raise a global Noether symmetry of the self- and anti-self-dual constituents into a local one, but for an effective composite system, consisting of the dual components and an interference term. The objective in Ref. [18] is to systemize the procedure like an algorithm and, consequently, to define the soldered action.

An iterative Noether procedure was adopted in Ref. [18] to lift the global symmetries. Therefore assume that the symmetries in question are being described by the local actions $S_{ \pm}\left(\phi_{ \pm}^{\eta}\right)$, invariant under a global multiparametric transformation

$$
\delta \phi_{ \pm}^{\eta}=\alpha^{\eta}
$$

where $\eta$ represents the tensorial character of the basic fields in the dual actions $S_{ \pm}$and, for notational simplicity, will be dropped from now on. As it is well known, we can write

$$
\delta S_{ \pm}=J^{ \pm} \partial_{ \pm} \alpha
$$

where $J^{ \pm}$are the Noether currents.

Now, under local transformations these actions will not remain invariant, and Noether counterterms become necessary to reestablish the invariance, along with appropriate auxiliary fields $B^{(N)}$, the so-called soldering fields which have no dynamics. Nevertheless, we can say that $B^{(N)}$ is an auxiliary field which makes a wider range of gauge-fixing conditions available [21]. In this way, the $N$ action can be written as

$$
S_{ \pm}\left(\phi_{ \pm}\right)^{(0)} \rightarrow S_{ \pm}\left(\phi_{ \pm}\right)^{(N)}=S_{ \pm}\left(\phi_{ \pm}\right)^{(N-1)}-B^{(N)} J_{ \pm}^{(N)} .
$$

Here $J_{ \pm}^{(N)}$ are the $N$-iteration Noether currents. For the selfand anti-self-dual systems we have in mind that this iterative gauging procedure is (intentionally) constructed not to produce invariant actions for any finite number of steps. However, if after $N$ repetitions, the noninvariant piece ends up being only dependent on the gauging parameters, but not on the original fields, there will exist the possibility of mutual cancelation if both self- and anti-self-gauged systems are put together. Then, suppose that after $N$ repetitions we arrive at the following simultaneous conditions:

$$
\begin{array}{r}
\delta S_{ \pm}\left(\phi_{ \pm}\right)^{(N)} \neq 0 \\
\delta S_{B}\left(\phi_{ \pm}\right)=0
\end{array}
$$

with $S_{B}$ being the so-called soldered action,

$$
S_{B}\left(\phi_{ \pm}\right)=S_{+}^{(N)}\left(\phi_{+}\right)+S_{-}^{(N)}\left(\phi_{-}\right)+\text {contact terms }
$$

where the contact terms are generally quadratic functions of the soldering fields. Then we can immediately identify the (soldering) interference term as

$$
S_{\text {int }}=\text { contact terms }-\sum_{N} B^{(N)} J_{ \pm}^{(N)}
$$

Incidentally, these auxiliary fields $B^{(N)}$ may be eliminated, for instance, through their equations of motion, from the resulting effective action, in favor of the physically relevant degrees of freedom. It is important to notice that after the elimination of the soldering fields, the resulting effective action will not depend on either self- or anti-self-dual fields $\phi_{ \pm}$ but only in some collective field, say $\Phi$, defined in terms of the original ones in a (Noether) invariant way,

$$
S_{B}\left(\phi_{ \pm}\right) \rightarrow S_{e f f}(\Phi)
$$

Analyzing in terms of the classical degrees of freedom, it is obvious that we have now a bigger theory. Once such effective action has been established, the physical consequences of the soldering are readily obtained by simple inspection. This will progressively be clarified in the specific application to be given next.

In order to present an example, we will analyze the Siegel chiral actions in the light of the interference phenomenon. ${ }^{3}$ First of all, we have to describe the light-front variables used in this paper as

$$
\begin{aligned}
& x_{ \pm}=\frac{1}{\sqrt{2}}\left(x_{0} \pm x_{1}\right), \\
& \partial_{ \pm}=\frac{1}{\sqrt{2}}\left(\partial_{0} \pm \partial_{1}\right), \\
& A_{ \pm}=\frac{1}{\sqrt{2}}\left(A_{0} \pm A_{1}\right),
\end{aligned}
$$

and now we can work out our example.

\section{A. An example: The Siegel action}

The original classical Lagrangian density for a chiral scalar field as introduced by Siegel [1] for a left moving scalar (a lefton) is [35]

\footnotetext{
${ }^{3} \mathrm{We}$ will follow the steps given in Ref. [19].
} 


$$
\begin{aligned}
\mathcal{L}_{0}^{(+)} & =\partial_{+} \varphi \partial_{-} \varphi+\lambda_{++} \partial_{-} \varphi \partial_{-} \varphi \\
& =\frac{1}{2} \sqrt{g} g^{\alpha \beta} \partial_{\alpha} \varphi \partial_{\beta} \varphi
\end{aligned}
$$

where the metric is given by

$$
\begin{aligned}
& g^{++}=0, \quad g^{+-}=1, \\
& g^{--}=2 \lambda_{++} .
\end{aligned}
$$

The Lagrangian (9) is invariant under Siegel gauge symmetry which is an invariance under the combined coordinate transformation and a Weyl rescaling of the form

$$
\begin{gathered}
x_{-} \rightarrow \tilde{x}_{-}=x_{-}-\epsilon\left(x_{+}, x_{-}\right), \\
\delta_{w} g_{\alpha \beta}=-g_{\alpha \beta} \partial_{-} \epsilon^{-},
\end{gathered}
$$

where $\epsilon^{ \pm}=\epsilon^{ \pm}\left(x_{ \pm}\right)$.

The fields $\varphi$ and $\lambda_{++}$transform under Eq. (11) as follows:

$$
\begin{aligned}
\delta \varphi & =\epsilon^{-} \partial_{-} \varphi \\
\delta \lambda_{++} & =-\partial_{+} \epsilon+\epsilon \partial_{+} \lambda_{++}-\lambda_{++} \partial_{+} \epsilon^{-} .
\end{aligned}
$$

In addition, Eq. (9) is invariant under the global axial transformation

$$
\varphi \rightarrow \tilde{\varphi}=\varphi+\bar{\varphi}
$$

where we have currents associated with this axial symmetry. It is beyond the scope of our work to write explicitly these axial currents as well as the conserved vector current. These objects can be found in the literature (see Ref. [35], for example).

The symmetry (12) describes a lefton. This is the main difference between a lefton (righton) and a left-moving (right-moving) FJ particle. The first is provided with symmetry and dynamics, while the second is responsible only for the dynamics of the theory. We can also say that the lefton (or righton) carries the anomaly of the system [26] (the wellknown Siegel anomaly), since it is relative to the symmetry of the theory.

Similarly, one can gauge the semilocal affine symmetry

$$
\begin{aligned}
\delta \varphi & =\epsilon^{+} \partial_{+} \varphi \\
\delta \lambda_{--} & =-\partial_{-} \epsilon^{+}+\epsilon^{+} \partial_{+} \lambda_{--}-\lambda_{--} \partial_{+} \epsilon^{+}
\end{aligned}
$$

to obtain the righton. Next we will promote the fusion of the righton and the lefton obtaining the final soldered action.

\section{B. The soldering procedure}

In fact, if we construct the righton and lefton chiral boson actions as

$$
\mathcal{L}_{0}^{( \pm)}=\frac{1}{2} J_{ \pm}(\varphi) \partial_{\mp} \varphi
$$

with

$$
J_{ \pm}(\varphi)=2\left(\partial_{ \pm} \varphi+\lambda_{ \pm \pm} \partial_{\mp} \varphi\right),
$$

it is easy to verify that these models are indeed invariant under Siegel's transformations (12) and (14), using that

$$
\delta J_{ \pm}=\epsilon_{ \pm} \partial_{\overline{+}} J_{ \pm} .
$$

It is worth mentioning at this point that Siegel's actions for leftons and rightons can be seen as the action for a scalar field immersed in a gravitational background whose metric is appropriately truncated. In this sense, Siegel symmetry for each chirality can be seen as a truncation of the reparametrization symmetry existing for the scalar field action. We should mention that the Noether current $J_{+}$defined above is in fact the nonvanishing component of the left chiral current $J_{+}=J_{(L)}^{-}$, while $J_{-}$is the nonvanishing component of the right chiral current $J_{-}=J_{(R)}^{+}$, with the left and right currents being defined in terms of the axial and vector currents as

$$
\begin{aligned}
& J_{\mu}^{(L)}=J_{\mu}^{(A)}+J_{\mu}^{(V)}, \\
& J_{\mu}^{(R)}=J_{\mu}^{(A)}-J_{\mu}^{(V)} .
\end{aligned}
$$

Let us next consider the question of the vector gauge symmetry. We can use the iterative Noether procedure described above to gauge the global U(1) symmetry,

$$
\begin{aligned}
\delta \varphi & =\alpha, \\
\delta \lambda_{++} & =0,
\end{aligned}
$$

possessed by Siegel's model (9). Under the action of the group of transformations (19), written now as a local parameter, the action (9) changes as

$$
\delta \mathcal{L}_{0}^{(+)}=\partial_{-} \alpha J_{+}
$$

with the Noether current $J_{+}=J_{+}(\varphi)$ being given as in Eq. (16). To cancel out this piece, we introduce the soldering field $B_{-}$coupled to the Noether current, redefining the original Siegel's Lagrangian density as

$$
\mathcal{L}_{0}^{(+)} \rightarrow \mathcal{L}_{1}^{(+)}=\mathcal{L}_{0}^{(+)}+B_{-} J_{+},
$$

where the variation of the gauge field is defined conveniently as 


$$
\delta B_{-}=-\partial_{-} \alpha
$$

As the variation of $\mathcal{L}_{1}^{(+)}$does not vanish up to total derivatives, we introduce a further modification as

$$
\mathcal{L}_{1}^{(+)} \rightarrow \mathcal{L}_{2}^{(+)}=\mathcal{L}_{1}^{(+)}+\lambda_{++} B_{-}^{2}
$$

whose variation gives

$$
\delta \mathcal{L}_{2}^{(+)}=2 B_{-} \partial_{+} \alpha .
$$

This piece cannot be canceled by a Noether counterterm, so that a gauge invariant action for $\varphi$ and $B_{-}$does not exist, at least with the introduction of only one gauge field. We observe, however, that this action has the virtue of having a variation dependent only on $B_{-}$and $\alpha$, and not on $\varphi$. Expression (24) is a reflection of the standard anomaly ${ }^{4}$ that is intimately connected with the chiral properties of $\varphi$.

Now, if the same gauging procedure is followed for a Siegel boson of opposite chirality, say

$$
\mathcal{L}_{0}^{(-)}=\partial_{+} \rho \partial_{-} \rho+\lambda_{--} \partial_{+} \rho \partial_{+} \rho,
$$

subject to

$$
\begin{gathered}
\delta \rho=\alpha, \\
\delta \lambda_{--}=0, \\
\delta B_{+}=-\partial_{+} \alpha,
\end{gathered}
$$

then one finds that the sum of the right and left gauged actions $\mathcal{L}_{2}^{(+)}+\mathcal{L}_{2}^{(-)}$can be made gauge invariant if a contact term of the form

$$
\mathcal{L}_{C}=2 B_{+} B_{-}
$$

is introduced. One can check that indeed the complete gauged Lagrangian

$$
\begin{aligned}
\mathcal{L}_{T O T}= & \partial_{+} \varphi \partial_{-} \varphi+\lambda_{++} \partial_{-} \varphi \partial_{-} \varphi+\partial_{+} \rho \partial_{-} \rho+\lambda_{--} \partial_{+} \rho \partial_{+} \rho \\
& +B_{+} J_{-}(\rho)+B_{-} J_{+}(\varphi)+\lambda_{--} B_{+}^{2}+\lambda_{++} B_{-}^{2} \\
& +2 B_{-} B_{+}
\end{aligned}
$$

with $J_{ \pm}$defined in Eq.(16) above, is invariant under the set of transformations (19), (22), and (26). For completeness, we note that Lagrangian (28) can also be written in the form

$$
\begin{aligned}
\mathcal{L}_{T O T}= & D_{+} \varphi D_{-} \varphi+\lambda_{++} D_{-} \varphi D_{-} \varphi+D_{+} \rho D_{-} \rho \\
& +\lambda_{--} D_{+} \rho D_{+} \rho+(\varphi-\rho) E,
\end{aligned}
$$

up to total derivatives. In the above expression, we have introduced the covariant derivatives $D_{ \pm} \varphi=\partial_{ \pm} \varphi+B_{ \pm}$, with

\footnotetext{
${ }^{4}$ The soldering analysis of the anomaly has been depicted in Ref. [37].
}

a similar expression for $D_{ \pm} \rho$, and $E \equiv \partial_{+} B_{-}-\partial_{-} B_{+}$. In form (29), $\mathcal{L}_{\text {TOT }}$ is manifestly gauge invariant.

After solving the equations of motion for the soldering fields we can write

$$
\mathcal{L}_{g}=\frac{1}{2} \sqrt{-g} g{ }^{\alpha \beta} \partial_{\alpha} \Phi \partial_{\beta} \Phi,
$$

where in the above expression we have introduced the metric tensor density

$$
\begin{aligned}
& \sqrt{-g} g^{--}=-4 \frac{\lambda_{++}}{\Delta}, \\
& \sqrt{-g} g^{++}=-4 \frac{\lambda_{--}}{\Delta}, \\
& \sqrt{-g} g^{+-}=-\frac{2}{\Delta}\left(1+\lambda_{++} \lambda_{--}\right),
\end{aligned}
$$

where $\Delta=2\left(\lambda_{++} \lambda_{--}-1\right)$ and

$$
\Phi=\frac{1}{\sqrt{2}}(\rho-\varphi) .
$$

We observe that in two dimensions $\sqrt{-g} g^{\alpha \beta}$ needs only two parameters to be defined in a proper way. As it should be, $\operatorname{det}\left(\sqrt{-g} g^{\alpha \beta}\right)=-1$. We also note that, because of conformal invariance, we cannot determine $g_{\alpha \beta}$ itself. We could therefore think of $\mathcal{L}_{T O T}$ as an effective theory, which represents a scalar boson $\Phi$ in a gravitational background. It can be shown [19] that the action (30) can be made invariant under the full group of diffeomorphism. Hence we can easily see that, in terms of symmetry, the new theory is bigger than the old one. This new theory can be interpreted as a constructive interference of symmetries. However, solving the equations of motion for the multipliers, we can see that, in fact, this field has no dynamics. This characterizes a nonmover field, a noton, introduced by Hull [27] to cancel out the gravitational anomaly of the Siegel model.

\section{THE MASTER ACTION}

In this section we will propose a master action which represents, as a function of arbitrary parameters, several theories for the Siegel gauged model. In the second part we have accomplished the soldering of opposite chiral versions of this master action and applied the final result, i.e., the soldered action, on several models for the self-dual theory to make an interference analysis of the covariance of the new theories.

\section{A. The generalized gauged Siegel model}

Let us now construct a class of generalized actions for Abelian chiral bosons coupled to a gauge field for each 
chirality, i.e., for the coupled leftons $\left(\mathcal{L}_{L}\right)$ and rightons $\left(\mathcal{L}_{R}\right)$. We will call it the generalized gauged Siegel model (GGSM):

$$
\mathcal{L}_{L}^{(0)}=\left(\partial_{+} \phi+a_{1} A_{+}\right)\left(\partial_{-} \phi+a_{2} A_{-}\right)+\lambda_{++}\left(\partial_{-} \phi+a_{3} A_{-}\right)^{2},
$$

$\mathcal{L}_{R}^{(0)}=\left(\partial_{+} \rho+b_{1} A_{+}\right)\left(\partial_{-} \rho+b_{2} A_{-}\right)+\lambda_{--}\left(\partial_{+} \rho+b_{3} A_{+}\right)^{2}$,

where $a_{i}, b_{i}(i=1,2,3)$ are parameters that define the theory studied and $A_{ \pm}$are the vector field components. We will see below that making simple substitutions of these parameters we can obtain several gauged forms of the Siegel theory that appear in the literature. It is important to observe the difference between the vector fields $A_{ \pm}$above and the soldering fields $B_{ \pm}$of Eqs. (29). The $A$ fields are external (or background) fields and hence one does not consider the variation (and extrema) of the actions under the variations of these fields. The last are the auxiliary fields, as mentioned above, which help in the soldering process and will be naturally eliminated by solving their equations of motion.

Following the steps of the soldering formalism studied in the last section, we can start considering the variation of the Lagrangians under the usual transformations,

$$
\delta \phi=\delta \rho=\alpha \quad \text { and } \quad \delta A_{\mu}=\partial_{\mu} \alpha
$$

where $\mu=+,-$. Now, consider that this symmetry is a global one with, obviously, a global parameter $\alpha$ so that the above transformations take the form

$$
\delta \phi=\delta \rho=\alpha \quad \text { and } \quad \delta A_{\mu}=0 .
$$

Remember that the soldering process consists in lifting the gauging of a global symmetry to its local version. Hence we will consider from now on the transformations (35) as local. Let us continue with the procedure writing only the main steps of the procedure.

In terms of the Noether currents we can construct

$$
\delta \mathcal{L}_{L, R}^{(0)}=J_{\phi, \rho}^{\mu} \partial_{\mu} \alpha
$$

where

$$
\begin{aligned}
& J_{\phi}^{+}=a_{2} A_{-}, \\
& J_{\phi}^{-}=2 \partial_{+} \phi+a_{1} A_{+}+2 \lambda_{++}\left(\partial_{-} \phi+a_{3} A_{-}\right), \\
& J_{\rho}^{+}=b_{2} A_{-}, \\
& J_{\rho}^{-}=2 \partial_{-} \rho+b_{2} A_{-}+2 \lambda_{--}\left(\partial_{+} \rho+b_{3} A_{+}\right) .
\end{aligned}
$$

The next iteration, as seen above, can be performed introducing auxiliary fields, the so-called soldering fields

$$
\mathcal{L}_{L, R}^{(1)}=\mathcal{L}_{L, R}^{(0)}-B_{\mu} J_{\phi, \rho}^{\mu},
$$

and one can easily see that the gauge variation of the GGSM is

$$
\delta \mathcal{L}_{L, R}^{(1)}=-B_{\mp} \delta B_{ \pm}-\lambda_{ \pm \pm} \delta B_{\mp}^{2} .
$$

Let us define the variation of $B_{ \pm}$as

$$
\delta B_{ \pm}=\partial_{ \pm} \alpha,
$$

and we see that the variation of $\mathcal{L}_{L, R}^{(1)}$ does not depend either on $\phi$ or $\rho$. Hence, as explained in the last section, we can construct the final (soldered) Lagrangian as

$$
\begin{aligned}
\mathcal{L}= & \mathcal{L}_{L} \oplus \mathcal{L}_{R} \\
= & \mathcal{L}_{L}^{(1)}+\mathcal{L}_{R}^{(1)}+2 B_{+} B_{-}+\lambda_{++} B_{-}^{2}+\lambda_{--} B_{+}^{2} \\
= & \left(\partial_{+} \phi+a_{1} A_{+}\right)\left(\partial_{-} \phi+a_{2} A_{-}\right)+\lambda_{++}\left(\partial_{-} \phi+a_{3} A_{-}\right)^{2} \\
& +\left(\partial_{+} \rho+b_{1} A_{+}\right)\left(\partial_{-} \rho+b_{2} A_{-}\right)+\lambda_{--}\left(\partial_{+} \rho+b_{3} A_{+}\right)^{2} \\
& -B_{\mu} J_{\phi}^{\mu}-B_{\mu} J_{\rho}^{\mu}+2 B_{+} B_{-}+\lambda_{++} B_{-}^{2}+\lambda_{--} B_{+}^{2},
\end{aligned}
$$

which remains invariant under the combined transformations (34) and (39). Following the steps of the algorithm depicted in the last section, we have to eliminate the soldering fields solving their equations of motion which results in

$$
B_{ \pm}=\frac{J^{\mp}-\lambda_{ \pm \pm} J^{ \pm}}{2(1-\lambda)},
$$

where $\lambda=\lambda_{++} \lambda_{--}$and $J^{ \pm}=J_{\phi}^{ \pm}+J_{\rho}^{ \pm}$.

Substituting it back in Eq. (41) we have the final soldered action

$$
\begin{aligned}
\mathcal{L}= & \frac{1}{2} \sqrt{-g} g^{\mu \nu} \partial_{\mu} \Phi \partial_{\nu} \Phi+\frac{1}{1-\lambda}\left\{\left(a_{1}+b_{1} \lambda-2 \lambda b_{3}\right) \partial_{-} \Phi A_{+}\right. \\
& +\left(2 \lambda a_{3}-a_{2} \lambda-b_{2}\right) \partial_{+} \Phi A_{-} \\
& +\lambda_{++}\left(2 a_{3}-a_{2}-b_{2}\right) \partial_{-} \Phi A_{-} \\
& +\lambda_{--}\left(a_{1}+b_{1}-2 b_{3}\right) \partial_{+} \Phi A_{+}+C_{1} \lambda_{++} A_{-}^{2} \\
& \left.+C_{2} \lambda_{--} A_{+}^{2}+C_{\lambda} A_{+} A_{-}\right\},
\end{aligned}
$$

where the new compound fields are defined as $\Phi=\phi-\rho$. The new parameters are

$$
\begin{aligned}
C_{1}= & a_{3}^{2}-b_{2} a_{3}+\frac{1}{4}\left(a_{2}+b_{2}\right)^{2}, \\
C_{2}= & b_{3}^{2}-b_{3} a_{1}+\frac{1}{4}\left(a_{1}+b_{1}\right)^{2}, \\
C_{\lambda}= & \left(\frac{1}{2}-\lambda\right) a_{1} a_{2}+\left(\frac{1}{2}-\lambda\right) b_{1} b_{2}-\frac{1}{2}\left(a_{1} b_{2}+b_{1} a_{2}\right) \\
& +\left[\left(a_{2}+b_{2}\right) b_{3}+\left(a_{1}+b_{1}\right) a_{3}-2 a_{3} b_{3}\right] \lambda-a_{2} a_{3} \lambda_{++} \\
& -b_{1} b_{3} \lambda_{--}
\end{aligned}
$$

and the metric is

$$
\frac{1}{2} \sqrt{-g} g^{\mu \nu}=\frac{1}{2(1-\lambda)}\left(\begin{array}{cc}
2 \lambda_{--} & 1+\lambda \\
1+\lambda & 2 \lambda_{++}
\end{array}\right)
$$


which reminds us of the gravitational feature of the soldered action of the two Siegel modes. We can note that the action (43) is covariant. Hence, in this case, we have that the covariance of the generalized gauged Siegel action is maintained. This general action form will allow us to apply it to the various gauged theories for the chiral boson with secondorder constraint. This will be accomplished next.

\section{B. The self-dual models}

In this section we will analyze five kinds of theories in the light of the soldering formalism. The first of them is the well-known Siegel's action [1], studied in Sec. II. It has been used to demonstrate the validity of the general soldered action (43). The second example, which is also not a new result, will be a coupling of the chiral boson with a gauge field. We are talking, in this case, about the Gates and Siegel gauged action [10]. The new results will appear with the next three models. We will use three models well known in the literature: the one derivative gauged model, the massless Bellucci, Golterman, and Petcher model, and the Frishman and Sonnenschein model.

\section{Siegel's model}

It is easy to see that to obtain the expression (9) we have to fix the parameters with the following values:

$$
a_{i}=b_{i}=0,
$$

where $i=1,2,3$. Hence substituting these values in the expression (43) it follows that

$$
\begin{aligned}
\mathcal{L}_{T O T}= & \frac{1}{1-\lambda}\left\{\left(1+\lambda_{++} \lambda_{--}\right) \partial_{-} \Phi \partial_{+} \Phi+\lambda_{++}\left(\partial_{-} \Phi\right)^{2}\right. \\
& \left.+\lambda_{--}\left(\partial_{+} \Phi\right)^{2}\right\} \\
= & \frac{1}{2} \sqrt{-g} g^{\mu \nu} \partial_{\mu} \Phi \partial_{\nu} \Phi
\end{aligned}
$$

where $\frac{1}{2} \sqrt{-g} g^{\mu \nu}$, from now on, is written as in Eq. (45). This action represents, naively, a scalar field immersed in a gravitational background. However, as we have stressed in Sec. II, this expression also represents the noton action.

\section{Gates and Siegel's model}

Gates and Siegel [10] have studied the interactions of leftons and rightons with external vector fields including the supersymmetric and the non-Abelian cases. The soldering of this model has been obtained already in Ref. [26], but as a further test for our GGSM, let us write

$$
\begin{gathered}
\mathcal{L}_{G S}^{\phi}=\left(\partial_{-} \phi+2 A_{-}\right)\left(\partial_{+} \phi\right)+\lambda_{++}\left(\partial_{-} \phi+A_{-}\right)^{2}, \\
\mathcal{L}_{G S}^{\rho}=\left(\partial_{+} \rho+2 A_{+}\right)\left(\partial_{-} \rho\right)+\lambda_{--}\left(\partial_{+} \rho+A_{+}\right)^{2},
\end{gathered}
$$

and the correspondence with Eq. (33a) is direct,

$$
a_{2}=b_{1}=2, \quad a_{1}=b_{2}=0, \quad a_{3}=b_{3}=1 .
$$

The soldered action is, using Eq. (43),

$$
\mathcal{L}_{T O T}=\frac{1}{2} \sqrt{-g} g^{\mu \nu} \partial_{\mu} \Phi \partial_{\nu} \Phi-2 A_{-} A_{+},
$$

confirming the result in Ref. [26]. We can note that the covariance has not been broken.

The physical meaning of Eq. (50) can be appreciated by eliminating the multipliers and using the symmetry induced by the soldering [32], showing that it represents the action for the noton. In fact, Eq. (50) is basically the action proposed by Hull [27] as a candidate for canceling the Siegel anomaly. This field carries a representation of the full diffeomorphism group [27] while its chiral (Siegel) component carries the representation of the chiral diffeomorphism. Observe the complete disappearance of the dynamical sector due to the destructive interference between the leftons and the rightons. This happens because we have introduced only one soldering field to deal with both the dynamics and the symmetry. To recover dynamics we need to separate these sectors and solder them independently, as stressed in Ref. [26].

\section{One derivative gauged model}

This gauged form was introduced in Ref. [15], where only one kind of derivative was gauged,

$$
\begin{aligned}
& \mathcal{L}_{O D}^{\phi}=\left(\partial_{+} \phi+2 e A_{+}\right)\left(\partial_{-} \phi\right)+\lambda_{++}\left(\partial_{-} \phi\right)^{2}, \\
& \mathcal{L}_{O D}^{\rho}=\left(\partial_{-} \rho+2 e A_{-}\right)\left(\partial_{+} \rho\right)+\lambda_{--}\left(\partial_{+} \rho\right)^{2} .
\end{aligned}
$$

Hence immediately we have the correspondence with Eqs. (33a) and (33b) through the choice

$$
a_{2}=a_{3}=0, \quad b_{1}=b_{3}=0, \quad a_{1}=b_{2}=2 e
$$

and

$$
\begin{aligned}
\mathcal{L}_{T O T}= & \frac{1}{2} \sqrt{-g} g^{\mu \nu} \partial_{\mu} \Phi \partial_{\nu} \Phi \\
& +\frac{1}{1-\lambda}\left[-2 e\left(\partial_{-} \Phi A_{+}-\partial_{+} \Phi A_{-}\right)\right. \\
& -2 e\left(\lambda_{++} \partial_{-} \Phi A_{-}-\lambda_{--} \partial_{+} \Phi A_{+}\right) \\
& \left.+e^{2}\left(\lambda_{++} A_{-}^{2}+\lambda_{--} A_{+}^{2}-2 A_{+} A_{-}\right)\right] .
\end{aligned}
$$

In this case we can note that the decoupling of the vector fields has not occurred.

The final action is explicitly covariant, showing that the soldering procedure did not provide the break of covariance. We can classify this case as constructive interference of covariances, since Eqs. (51) are covariant also. 
4. The gauged massless Bellucci, Golterman, and Petcher model

The form of this gauged chiral boson action is

$$
\begin{aligned}
& \mathcal{L}_{B G P}^{\phi}=\left(\partial_{+} \phi\right)\left(\partial_{-} \phi+e A_{-}\right)+\lambda_{++}\left(\partial_{-} \phi+e A_{-}\right)^{2}, \\
& \mathcal{L}_{B G P}^{\rho}=\left(\partial_{-} \rho\right)\left(\partial_{+} \rho+e A_{+}\right)+\lambda_{--}\left(\partial_{+} \rho+e A_{+}\right)^{2},
\end{aligned}
$$

hence

$$
a_{1}=b_{2}=0, \quad a_{2}=a_{3}=b_{1}=b_{3}=e
$$

and the final action reads

$$
\begin{aligned}
\mathcal{L}_{T O T}= & \frac{1}{2} \sqrt{-g} g^{\mu \nu} \partial_{\mu} \Phi \partial_{\nu} \Phi+\frac{1}{1-\lambda}\left[e \lambda\left(\partial_{+} \Phi A_{-}-\partial_{-} \Phi A_{+}\right)\right. \\
& +e \lambda_{++} \partial_{-} \Phi A_{-}-e \lambda_{--}(1+\lambda) \partial_{+} \Phi A_{+} \\
& +\frac{5}{4} e^{2}\left(\lambda_{++} A_{-}^{2}+\lambda_{--} A_{+}^{2}\right) \\
& \left.-e^{2}\left(\frac{1}{2}-\lambda_{++}-\lambda_{--}\right) A_{+} A_{-}\right] ;
\end{aligned}
$$

it is easy to see that last two terms break the covariance. Hence in this case we have clearly a destructive interference of covariances.

\section{The Frishman and Sonnenschein model}

The chiral actions developed in Ref. [35] are

$$
\begin{gathered}
\mathcal{L}_{F S}^{\phi}=\left(\partial_{+} \phi\right)\left(\partial_{-} \phi\right)+\lambda_{++}\left(\partial_{-} \phi\right)^{2}+\partial_{+} \phi A_{-}-\partial_{-} \phi A_{+}, \\
\mathcal{L}_{F S}^{\rho}=\left(\partial_{-} \rho\right)\left(\partial_{+} \rho\right)+\lambda_{--}\left(\partial_{+} \rho\right)^{2}+\partial_{-} \rho A_{+}-\partial_{+} \rho A_{-},
\end{gathered}
$$

and identifying the parameters

$$
a_{1}=b_{2}=-1, \quad a_{2}=b_{1}=1, \quad a_{3}=b_{3}=0,
$$

we can construct the soldered action as

$$
\mathcal{L}_{T O T}=\frac{1}{2} \sqrt{-g} g^{\mu \nu} \partial_{\mu} \Phi \partial_{\nu} \Phi+\epsilon^{\mu \nu} \partial_{\mu} \Phi A_{\nu}+\frac{1-2 \lambda}{1-\lambda} A_{+} A_{-},
$$

where $\epsilon^{+-}=1$.

Now we have a constructive interference of covariance, since the soldered action is explicitly covariant.

\section{THE LORENTZ INVARIANCE ANALYSIS}

Let us now fix conditions over the parameters in order to respect a Lorentz invariance. In other words, we mean that we will fix conditions such that the constraints valid in one inertial reference system are valid in the other one. To do this, we will perform the Lorentz rotation [15]. This will be done in the corresponding FJ version of the GGSM proposed above.
The generalized gauged Siegel model, as we already know, is

$$
\begin{aligned}
\mathcal{L}_{G G S M}= & \left(\partial_{+} \phi+k_{1} A_{+}\right)\left(\partial_{-} \phi+k_{2} A_{-}\right) \\
& +\lambda_{++}\left(\partial_{-} \phi+k_{3} A_{-}\right)^{2} .
\end{aligned}
$$

The canonical momentum conjugated to $\phi$ is

$$
\begin{aligned}
\pi_{\phi} & =\phi^{\prime}+\frac{1}{2} k_{1}\left(A_{0}+A_{1}\right)+\frac{1}{2}\left(k_{2}-k_{3}\right)\left(A_{0}-A_{1}\right) \\
& =\phi^{\prime}+\frac{1}{2}\left(k_{1}+k_{2}-2 k_{3}\right) A_{0}+\frac{1}{2}\left(k_{1}-k_{2}+2 k_{3}\right) A_{1},
\end{aligned}
$$

and this is the generalized chiral constraint.

Using the first-order formalism of Faddeev and Jackiw with this momentum, we can construct a first-order Lagrangian density,

$$
\begin{aligned}
\mathcal{L}_{G G S M}= & \dot{\phi} \phi^{\prime}-\phi^{\prime 2}+\frac{A_{0}^{2}}{2}\left(k_{1} k_{2}-k_{3}^{2}\right)-\frac{A_{1}^{2}}{2}\left(k_{1} k_{2}+k_{3}^{2}\right) \\
& +\frac{A_{0}^{2}}{2}\left[\left(k_{1}+k_{2}-2 k_{3}\right) \dot{\phi}-\left(k_{1}-k_{2}-2 k_{3}\right) \phi^{\prime}\right] \\
& +\frac{A_{1}^{2}}{2}\left[\left(k_{1}-k_{2}+2 k_{3}\right) \dot{\phi}-\left(k_{1}+k_{2}+2 k_{3}\right) \phi^{\prime}\right]
\end{aligned}
$$

which is a constrained one. To verify the Lorentz invariance we have to note if the constraints are preserved from one inertial reference system to the other. To do this we have to apply the Lorentz rotation on the generalized chiral constraint. Constructing the rotation matrices as

$$
\left(\begin{array}{c}
\pi \\
\phi^{\prime}
\end{array}\right) \rightarrow\left(\begin{array}{cc}
\cosh \varphi & \sinh \varphi \\
\sinh \varphi & \cosh \varphi
\end{array}\right)\left(\begin{array}{c}
\tilde{\pi} \\
\tilde{\phi}^{\prime}
\end{array}\right)
$$

and

$$
\left(\begin{array}{c}
A_{0} \\
A_{1}
\end{array}\right) \rightarrow\left(\begin{array}{cc}
\cosh \varphi & \sinh \varphi \\
\sinh \varphi & \cosh \varphi
\end{array}\right)\left(\begin{array}{c}
\widetilde{A}_{0} \\
\widetilde{A}_{1}
\end{array}\right)
$$

and we have relations between the old fields and the new (tilde) fields. Writing Eq. (61) in a convenient way,

$$
\pi_{\phi}=\phi^{\prime}+\frac{C_{1}}{2} A_{0}+\frac{C_{2}}{2} A_{1}
$$

where $C_{1}=k_{1}+k_{2}-2 k_{3}$ and $C_{2}=k_{1}-k_{2}+2 k_{3}$.

After a little algebra, where we have provided the substitution of Eqs. (63) and (64) in Eq. (65), we can write

$$
\begin{aligned}
& C_{1} \cosh \varphi+C_{2} \sinh \varphi=C_{1}(\cosh \varphi-\sinh \varphi), \\
& C_{1} \sinh \varphi+C_{2} \cosh \varphi=C_{2}(\cosh \varphi-\sinh \varphi)
\end{aligned}
$$


Solving this system we can say that the generalized chiral constraints are Lorentz invariant, if

$$
C_{1}=-C_{2}
$$

In other words, we can say that with this solution the constraint is independent of the reference system.

Solving Eq. (67) we have that

$$
k_{1}=0 .
$$

With this result, we conclude that we can only gauge terms with the same light-cone variables, i.e.,

$$
\mathcal{L}=\left(\partial_{+} \phi\right)\left(\partial_{-} \phi+k_{2} A_{-}\right)+\lambda_{++}\left(\partial_{-} \phi+k_{3} A_{-}\right)^{2},
$$

which corroborates the results for the gauging of the FJ model.

At this point it is interesting to remark that, in the original proposal of this method for verifying the relativistic invariance using the Lorentz rotation $[15,16]$, it was supposed that the invariance should be imposed, and this had led to some criticisms [36]. Now we can see that in this approach, in fact, there is no need of ad hoc impositions.

\section{THE CHIRAL SCHWINGER MODEL WITH GENERALIZED CONSTRAINT}

In Ref. [15], the Lorentz rotation technique was used in the bosonized form of the chiral Schwinger model with a generalized constraint

$$
\Omega=\pi_{\phi}-\alpha \phi^{\prime}
$$

imposed on the first-order Lagrangian to determinate conditions on $\alpha$ such that we have a Lorentz invariant final theory.

Now we will disclose the conditions on $\alpha$ in the soldered action in order to have a covariant model. To begin with, let us write both chiralities of the effective Lagrangian [15],

$$
\begin{aligned}
\mathcal{L}_{\alpha}^{\phi}= & -\alpha \dot{\phi} \phi^{\prime}-\frac{1}{2}\left(\alpha^{2}+1\right) \phi^{\prime 2}+(\alpha+1) e \phi^{\prime}\left(A_{0}-A_{1}\right) \\
& -\frac{1}{2} e^{2}\left(A_{0}-A_{1}\right)+\frac{a}{2} A_{\mu}^{2}, \\
\mathcal{L}_{\alpha}^{\rho}= & \alpha \dot{\rho} \rho^{\prime}-\frac{1}{2}\left(\alpha^{2}+1\right) \rho^{\prime 2}+(\alpha+1) e \rho^{\prime}\left(A_{0}+A_{1}\right) \\
& -\frac{1}{2} e^{2}\left(A_{0}+A_{1}\right)+\frac{b}{2} A_{\mu}^{2},
\end{aligned}
$$

where, in Ref. [15], to produce a Lorentz covariant theory, $\alpha$ is the solution of the equation

$$
\left(\alpha^{2}+1\right) \phi^{\prime}-\left(g_{1} \alpha+g_{2}\right) A_{0}-\left(g_{2} \alpha+g_{1}\right) A_{1}=0 .
$$

The parameters $g_{1}$ and $g_{2}$ are $g_{1}=g_{2}=e\left(g_{1}=-g_{2}=e\right)$ for the right (left)-handed chiral Schwinger model.
Now, to perform an interference analysis, we have to impose the gauge transformations

$$
\left(\begin{array}{l}
\phi \\
\rho
\end{array}\right) \rightarrow\left(\begin{array}{l}
\phi \\
\rho
\end{array}\right)+\xi\left(\begin{array}{l}
1 \\
1
\end{array}\right)
$$

which, following the soldering mechanism, has as Noether currents

$$
\begin{aligned}
& J_{\phi}^{0}=J_{\rho}^{0}=0, \\
& J_{\phi}^{1}=-2 \alpha \dot{\phi}-\left(\alpha^{2}+1\right) \phi^{\prime}+e(\alpha+1)\left(A_{0}-A_{1}\right), \\
& J_{\rho}^{1}=2 \alpha \dot{\rho}-\left(\alpha^{2}+1\right) \rho^{\prime}+e(\alpha-1)\left(A_{0}+A_{1}\right) .
\end{aligned}
$$

Introducing the soldering fields and eliminating them by solving their equations of motion and substituting back into the contact terms of the action, we have a final soldered action

$$
\begin{aligned}
\mathcal{L}_{\text {FINAL }}= & -\frac{1}{4}\left(\alpha^{2}+1\right) \Phi^{\prime 2}+\frac{\alpha^{2}}{\alpha^{2}+1} \dot{\Phi}^{2}+\frac{2 \alpha^{2} e}{\alpha^{2}+1} \dot{\Phi} A_{0} \\
& +e \alpha \Phi^{\prime} A_{1}-\frac{2 \alpha e}{\alpha^{2}+1} \dot{\Phi} A_{1}+e \Phi^{\prime} A_{0} \\
& +e^{2}\left[\frac{\alpha^{2}}{\alpha^{2}+1}+\frac{1}{2}(a+b)-1\right] A_{0}^{2} \\
& +e^{2}\left[\frac{1}{\alpha^{2}+1}+\frac{1}{2}(a+b)-1\right] A_{1}^{2}-\frac{2 \alpha e^{2}}{\alpha^{2}+1} A_{0} A_{1},
\end{aligned}
$$

remembering that $\Phi=\phi-\rho$, as usual. We can easily see that $\mathcal{L}_{\text {FINAL }}$ does not describe a constrained system. The soldering procedure has broken the constraint feature of the system. This fact is contrary to the feature of Eqs. (71a) and (71b), which are constrained Lagrangians. Hence we will ask which conditions $\alpha$ must obey in order to preserve the manifest covariance and consequently the Lorentz invariance.

\section{A. Manifest covariance}

To obtain the manifest covariance, it is easy to see that in Eq. (75) $\alpha$ have to satisfy the following set of equations:

$$
\begin{aligned}
\frac{1}{4}\left(\alpha^{2}+1\right) & =\frac{\alpha^{2}}{\alpha^{2}+1}, \\
\frac{2 \alpha^{2} e}{\alpha^{2}+1} & =-\alpha, \\
\frac{2 \alpha}{\alpha^{2}+1} & =-1,
\end{aligned}
$$




$$
\begin{gathered}
-\frac{\alpha^{2}}{\alpha^{2}+1}=\frac{1}{\alpha^{2}+1}, \\
\frac{\alpha}{\alpha^{2}+1}=0 .
\end{gathered}
$$

Analyzing the solution $\alpha=0$ of equation (76e) we can easily see that it is not compatible with equations (76a), (76c), and (76d). We can observe, for instance, that equation (76d) presents a complex solution also, $\alpha= \pm i$. Hence, our soldered action is not manifestly covariant at all. It is interesting to notice that the massive terms for the gauge fields of the actions (71a) and (71b) have not influenced the final result. The condition to impose covariance on the gauge fields terms, i.e., $\alpha=0$, at the same time breaks the covariance of the action independently of the gauge field massive terms. This result supplements the one found in [14], where the soldering of two massless chiral Schwinger models generates a massive particle.

\section{B. Lorentz invariance}

As we saw in the last section, the action (75) is not constrained. So, to impose conditions on $\alpha$ to verify if our final (soldered) action, Eq. (75), is Lorentz invariant through a Lorentz rotation, we have to make a direct comparison term by term. The first step is to rewrite the action (75) as

$$
\begin{aligned}
\mathcal{L}= & a_{1} \phi^{\prime 2}+a_{2} \dot{\phi}^{2}+a_{3} \dot{\phi} A_{0}+e \alpha \phi^{\prime} A_{1}+e \phi^{\prime} A_{0}+a_{5} A_{0}^{2} \\
& +a_{6} A_{1}^{2}+a_{7} A_{0} A_{1}
\end{aligned}
$$

where

$$
\begin{aligned}
& a_{1}=-\frac{1}{4}\left(\alpha^{2}+1\right), \\
& a_{2}=\frac{\alpha^{2}}{\alpha^{2}+1}, \\
& a_{3}=-\frac{2 \alpha^{2}}{\alpha^{2}+1}, \\
& a_{4}=-\frac{2 e \alpha^{2}}{\alpha^{2}+1}, \\
& a_{5}=e^{2}\left[\frac{\alpha^{2}}{\alpha^{2}+1}+\frac{1}{2}(a+b)-1\right], \\
& a_{6}=e^{2}\left[\frac{1}{\alpha^{2}+1}+\frac{1}{2}(a+b)-1\right], \\
& a_{7}=-\frac{2 e^{2} \alpha^{2}}{\alpha^{2}+1} .
\end{aligned}
$$

Following the Lorentz rotation procedure, we have to establish the matrix relations between the old and the new (tilde) fields through the construction of the Lorentz rotation matrix,

$$
\left(\begin{array}{c}
\Phi \\
\Phi^{\prime}
\end{array}\right) \rightarrow\left(\begin{array}{cc}
\cosh \theta & \sinh \theta \\
\sinh \theta & \cosh \theta
\end{array}\right)\left(\begin{array}{c}
\tilde{\Phi} \\
\widetilde{\Phi}^{\prime}
\end{array}\right)
$$

and for the gauge fields components, Eq. (64).

Finally, after these substitutions, the transformed Lagrangian is

$$
\begin{aligned}
\mathcal{L}_{\text {FINAL }}= & \left(a_{2} x^{2}+a_{1} y^{2}\right) \tilde{\phi}^{2}+\left(a_{2} y^{2}+a_{1} x^{2}\right) \widetilde{\phi}^{\prime 2} \\
& +2\left(a_{1}+a_{2}\right) x y \tilde{\dot{\phi}} \widetilde{\phi}^{\prime} \\
& +\left(a_{3} y^{2}+e y^{2}+e \alpha x y+a_{4} x^{2}\right) \tilde{\dot{\phi}} \widetilde{A}_{0}+\left(a_{3} x^{2}+e x^{2}\right. \\
& \left.+e \alpha x y a_{4} y^{2}\right) \widetilde{\phi}^{\prime} \widetilde{A}_{1}+\left[e \alpha y^{2}+\left(a_{3}+e+a_{4}\right) x y\right] \tilde{\dot{\phi}} \widetilde{A}_{1} \\
& +\left[e \alpha x^{2}+\left(a_{3}+e+a_{4}\right) x y\right] \widetilde{\phi}^{\prime} \widetilde{A}_{0}+\left(a_{5} y^{2}+a_{7} x y\right. \\
& \left.+a_{6} x^{2}\right) \widetilde{A}_{0}^{2}+\left(a_{5} x^{2}+a_{7} x y+a_{6} y^{2}\right) \widetilde{A}_{1}^{2}+\left[\left(a _ { 7 } \left(y^{2}\right.\right.\right. \\
& \left.\left.+x^{2}\right)+2\left(a_{5}+a_{6}\right) x y\right] \widetilde{A}_{0} \widetilde{A}_{1},
\end{aligned}
$$

where $x=\sinh \theta$ and $y=\cosh \theta$.

We can notice the appearance of a $\tilde{\dot{\phi}} \widetilde{\phi}^{\prime}$ term. It does not exist in the action (75). So, it has to disappear. Then, we must have $a_{1}=-a_{2}$. Hence

$$
\frac{1}{4}\left(\alpha^{2}+1\right)=\frac{\alpha^{2}}{\alpha^{2}+1}
$$

and the solution is

$$
\alpha= \pm 1
$$

Substituting these values in Eq. (78) we can easily see that we cannot reproduce the action (75). So, the relativistic invariance, in the soldering procedure, has been broken. We have now a case of destructive interference of relativistic invariance.

\section{CONCLUSIONS}

In this work we have proposed a generalized gauged Siegel model (master action), which can represent some gauged actions depending on the choice of the parameters. We have promoted the fusion of two GGSM of opposite chiralities and obtained a soldered action. The application of this action to several gauged models already present in the literature showed new results, which can never be obtained by a naive addition of the classical Lagrangians.

Using the Lorentz rotation to test the relativistic invariance of this master action we have fixed one of the parameters, showing that, to keep the equivalence between the constraints in the two inertial reference systems, only one of the derivatives must be gauged. This is a new result about the 
issue of the chiral bosons coupled to gauge fields.

We have used the soldering formalism also to study the action developed in Ref. [15]. In a first step of the procedure, we have developed a soldered action, which brings both chiralities together. In order to keep the manifest covariance of this action, we have demonstrated that it is not possible to find the parameter such that we have a covariant theory showing a destructive interference of covariances. Interestingly we have found that the gauge field massive terms have not interfered in the process. Hence we have looked for a value that maintains the Lorentz invariance of the con- straints. The result confirms the one encountered in each chirality separately.

\section{ACKNOWLEDGMENTS}

The authors would like to thank C. Wotzasek and D. Dalmazi for useful conversations. E.M.C.A. is financially supported by Fundação de Amparo à Pesquisa do Estado de São Paulo (FAPESP). This work was partially supported by Conselho Nacional de Pesquisa e Desenvolvimento ( $\mathrm{CNPq})$. FAPESP and $\mathrm{CNPq}$ are Brazilian research agencies.
[1] W. Siegel, Nucl. Phys. B238, 307 (1984).

[2] R. Floreanini and R. Jackiw, Phys. Rev. Lett. 59, 1873 (1987).

[3] N. Marcus and J. Schwarz, Phys. Lett. 115B, 111 (1982); D. J. Gross, J. A. Harvey, E. Martinec, and R. Rhom, Phys. Rev. Lett. 54, 502 (1985).

[4] M. Gomes, V. Kurak, V. O. Rivelles, and A. J. da Silva, Phys. Rev. D 38, 1344 (1988).

[5] X. G. Wen, Phys. Rev. Lett. 64, 2206 (1990); Phys. Rev. B 41, 12838 (1990); M. Stone, ibid. 42, 212 (1990).

[6] H. O. Girotti, M. Gomes, V. Kurak, V. O. Rivelles, and A. J. da Silva, Phys. Rev. Lett. 60, 1913 (1988).

[7] S. Bellucci, R. Brooks, and J. Sonnenschein, Nucl. Phys. B304, 173 (1988).

[8] S. Bellucci, M. F. L. Golterman, and D. N. Petcher, Nucl. Phys. B326, 307 (1989); J. Sonnenschein, ibid. B309, 752 (1988).

[9] K. Harada, Phys. Rev. Lett. 64, 139 (1990).

[10] S. J. Gates, Jr. and W. Siegel, Phys. Lett. B 206, 631 (1988).

[11] S. Bellucci, R. Brooks, and J. Sonnenschein, Mod. Phys. Lett. A 3, 1537 (1988); S. Bellucci, M. F. L. Golterman, and D. N. Petcher, Phys. Lett. B 217, 289 (1989); S. Bellucci, D. A. Depireux, and S. James Gates, Jr., ibid. 232, 67 (1989); S. Bellucci, E. Ivanov, and S. Krivonos, Ann. Phys. (N.Y.) 217, 194 (1992).

[12] M. Stone, Illinois Report ILL-(TH)-89-23, 1989; Phys. Rev. Lett. 63, 731 (1989); Nucl. Phys. B327, 399 (1989).

[13] E. Witten, Commun. Math. Phys. 92, 455 (1984).

[14] E. M. C. Abreu, R. Banerjee, and C. Wotzasek, Nucl. Phys. B509, 519 (1998).

[15] S. A. Dias and A. de Souza Dutra, Phys. Lett. B 293, 132 (1992).
[16] A. de Souza Dutra, Mod. Phys. Lett. A 7, 1449 (1992).

[17] D. Bazeia, Mod. Phys. Lett. A 5, 2447 (1990).

[18] For a review, see C. Wotzasek, 'Soldering formalism: Theory and applications,'” hep-th/9806005.

[19] R. Amorim, A. Das, and C. Wotzasek, Phys. Rev. D 53, 5810 (1996).

[20] A. Pressley and G. Segal, Loup Groups (Oxford University Press, Oxford, 1986).

[21] K. Harada, Int. J. Mod. Phys. A 6, 3399 (1991).

[22] R. Jackiw and R. Rajaraman, Phys. Rev. Lett. 54, 1219 (1985).

[23] R. Jackiw, Diverse Topics in Theoretical and Mathematical Physics (World Scientific, Singapore, 1995).

[24] R. Banerjee and C. Wotzasek, "Soldering formalism and mass generation in four dimensions," hep-th/0004191.

[25] A. de Souza Dutra, Phys. Lett. B 286, 285 (1992).

[26] E. M. C. Abreu and C. Wotzasek, Phys. Rev. D 58, 101701 (1998).

[27] C. M. Hull, Phys. Lett. B 206, 234 (1988); 212, 437 (1988).

[28] P. K. Townsend, K. Pilch, and P. van Niewwenhuizen, Phys. Lett. 136B, 452 (1984).

[29] S. Deser and R. Jackiw, Phys. Lett. 139B, 371 (1984).

[30] R. Banerjee and C. Wotzasek, Nucl. Phys. B527, 402 (1998).

[31] J. Schwarz and A. Sen, Nucl. Phys. B411, 35 (1994).

[32] C. Wotzasek, Phys. Rev. D 57, 4990 (1998).

[33] E. M. C. Abreu, A. Ilha, C. Neves, and C. Wotzasek, Phys. Rev. D 61, 025014 (2000).

[34] P. Mitra, Phys. Lett. B 284, 23 (1992).

[35] Y. Frishman and J. Sonnenschein, Nucl. Phys. B301, 346 (1998).

[36] C. Wotzasek, Phys. Lett. B 324, 28 (1994).

[37] R. Amorim and A. Das, Phys. Rev. D 54, 4177 (1996). 